1 Universidade Federal de São João del-Rei (UFSJ) Divinópolis (MG), Brasil. enftarcisio@ufsj.edu.br

2 Universidade Federal de São João del-Rei (UFSJ) Divinópolis (MG), Brasil. annagsd@hotmail.com

3 Universidade Federal de São João del-Rei (UFSJ) Divinópolis (MG), Brasil. elietealbano@hotmail.com

${ }^{4}$ Secretaria Municipal de Saúde de Divinópolis Divinópolis (MG), Brasil. josyds1@yahoo.com.br

\section{Avaliação da atenção primária: o ponto de vista de usuários}

\author{
Evaluation of primary care: the point of view of users \\ Tarcísio Laerte Gontijo', Anna Gabryela Sousa Duarte2 ${ }^{2}$ Eliete Albano de Azevedo Guimarães ${ }^{\mathbf{3}}$, \\ Joseane da Silva 4
}

RESUMO O objetivo deste artigo foi avaliar atributos da Atenção Primária à Saúde (APS), sob a percepção de usuários. Trata-se de estudo transversal que teve como participantes 384 usuários adultos vinculados a equipes de APS. Aplicou-se o instrumento Primary Care Assessment Tool - usuários adultos, versão reduzida. O Grau de Afiliação e dois atributos atingiram escores médios superiores a 6,6, sendo assim, bem avaliados. Os demais obtiveram escores inferiores a 6,6, não atingindo a média estabelecida como desejável. Conclui-se que os atributos da APS analisados receberam avaliação heterogênea e que a maioria necessita de um olhar mais atento e de investimento por parte dos gestores e profissionais.

PALAVRAS-CHAVE Avaliação de serviços de saúde. Atenção Primária à Saúde. Serviços de saúde.

ABSTRACT The aim of this paper was to evaluate attributes of the Primary Health Care (PHC), under the perception of users. It is a cross-sectional study that had as participants 384 adult users linked to PHC teams. The Primary Care Assessment Tool - adult users, reduced version was applied. The Degree of Affiliation and two attributes reached average scores higher than 6.6 and were thus well evaluated. The other attributes had scores lower than 6.6, not reaching the average score established as desirable. It is concluded that the analyzed APS attributes received a heterogeneous evaluation and that most of them need a closer look and investment by the managers and professionals.

KEYWORDS Health services research. Primary Health Care. Health services. 


\section{Introdução}

A Atenção Primária à Saúde (APS) caracteriza-se por um conjunto de ações de caráter individual e coletivo voltado para a promoção e proteção da saúde, prevenção dos agravos, diagnóstico, tratamento, reabilitação, redução de danos e manutenção da saúde (BRASIL, 2012). Ela é considerada como ordenadora de um sistema, principal porta de entrada dos usuários ao sistema de saúde e centro de comunicação com outros pontos que integram as redes de atenção (BRASIL, 2012).

Dentre os fundamentos e diretrizes da APS, destacam-se: ter território adscrito, acesso universal e contínuo a serviços de saúde de qualidade e resolutivos, desenvolvimento de vínculo e responsabilização, coordenação da atenção integrada e continuada centrada no indivíduo e na família e estímulo à participação comunitária (BRASIL, 2012; SILVA ET AL., 2014).

No Brasil, há modelos distintos de organização da APS em desenvolvimento nas diferentes regiões do País, em função de interesses, capacidade de gestão e concepções distintas, sendo mais prevalentes a Estratégia Saúde da Família (ESF) e a atenção primária tradicional (PAIM, 2012). Segundo dados do Ministério da Saúde (MS), a ESF em julho de 2016, atingia uma cobertura de $62,8 \%$ da população brasileira, contando com 41.370 equipes implantadas (BRASIL, 2017).

Em relação às especificidades da ESF, faz-se necessária a existência de equipe multiprofissional composta por, no mínimo, médico e enfermeiro generalistas, auxiliar ou técnico de enfermagem e agentes comunitários de saúde, podendo-se acrescentar a essa composição os profissionais de saúde bucal (BRASIL, 2012). Já a estratégia tradicional conta com centros de saúde equipados com médicos de diversas especialidades básicas, enfermeiros, auxiliares de enfermagem, dentistas e pessoal de apoio técnico, atende a uma demanda espontânea e/ou encaminhada por outros serviços (SALA ET AL., 2011).
A relevância dos serviços de APS e a compreensão das ações, programas e políticas implantadas, como também os efeitos dessas intervenções, requerem avaliações permanentes, a fim de manter e/ou melhorar os resultados e a qualidade da assistência prestada (OLIVEIRA ET AL., 2013; CAMPOS ET AL., 2014; CARNEIRO ET AL., 2014).

No estabelecimento de um marco de referência para a avaliação de serviços da APS, conceituam-se quatro atributos essenciais: acesso de primeiro contato, que se define como a acessibilidade e uso do serviço a cada novo problema ou novo episódio de um problema; longitudinalidade, que pressupõe a existência de uma fonte continuada de atenção e seu uso ao longo do tempo; integralidade, que implica no leque de serviços disponíveis e prestados e nas ações que o serviço de saúde deve oferecer para que os usuários recebam atenção integral; e coordenação da atenção, que pressupõe alguma forma de continuidade da atenção, além do reconhecimento de problemas e serviços para o atendimento das necessidades atuais (STARFIELD; XU; SHI, 2001).

Além desses atributos essenciais, estabelecem-se outras três características, denominadas atributos derivados: orientação familiar, que considera o contexto familiar na atenção integral; orientação comunitária, que procede no reconhecimento das necessidades em saúde da comunidade; e competência cultural, que envolve a atenção às características culturais especiais (STARFIELD; $\mathrm{XU}$; SHI, 2001).

Todos esses atributos podem ser avaliados separadamente, apesar de se apresentarem intimamente inter-relacionados na prática assistencial, individual ou coletiva. Além disso, representam importantes indicadores de qualidade da APS, sendo que quanto maior for a presença e a força desses atributos, mais forte é a sua orientação para a APS (ARAÚJO, 2015; STARFIELD; XU; SHI, 2001; SILVA ET AL., 2014).

Existem evidências relacionadas com o impacto positivo da APS em países em 
desenvolvimento, além da associação entre o maior grau de orientação à APS e o aumento da efetividade dos sistemas de saúde, satisfação dos usuários, promoção da equidade, integralidade e eficiência (SILVA ET AL., 2014). Usuários indicam uma boa qualidade geral da APS, sendo os atributos essenciais longitudinalidade e coordenação do cuidado os que mais se destacam na avaliação (HARZHEIM ET AL., 2016).

Na busca por avaliações de desempenho da APS, a opinião, o interesse e o envolvimento dos usuários são imprescindíveis. Apesar de estudos incentivados pelo MS, as avaliações dos serviços de saúde a fim de nortear a elaboração e a reorientação de políticas e programas na APS, segundo a percepção de usuários, são consideradas incipientes e um grande desafio para gestores; além disso, não estão disponíveis instrumentos de fácil aplicação para uso rotineiro (CAMPOS ET AL., 2014).

Os processos de avaliação sob percepção de usuários podem atuar para a construção de uma nova perspectiva do cuidado, além de monitorar as atividades, fortalecer o controle social e a participação/inclusão dos usuários nos processos de planejamento (BRANDÃO; GIOVANELLA; CAMPOS, 2013).

Nessa perspectiva, o presente estudo teve por objetivo avaliar os atributos da APS, sob percepção de usuários de um município da região centro-oeste de Minas Gerais (MG).

\section{Métodos}

Trata-se de estudo de abordagem quantitativa com delineamento transversal realizado em um município da região centro-oeste de Minas Gerais no ano de 2016. Esse município possui população estimada de aproximadamente 232.945 habitantes (IBGE, 2016), situa-se entre os 10 principais do estado e conta com 42 Unidades de Atenção Primária à Saúde (Uaps), sendo 32 equipadas com equipes de ESF e 10 com equipes de atenção primária tradicional. A cobertura da população pela ESF é de $44 \%$; e o restante, por unidades tradicionais.

Foram incluídos, como participantes, usuários de Uaps. Das 42 unidades existentes, 4 foram excluídas por estarem localizadas em zona rural e 12 por terem menos de um ano de implantação. Assim, abordaram-se usuários de 26 equipes de APS, sendo 16 equipes de ESF e 10 equipes de atenção primária tradicional, as quais atendem a uma população de 133.047 habitantes. O dimensionamento amostral dos entrevistados foi realizado definindo-se uma população finita e um erro absoluto de $5 \%$. O resultado do dimensionamento demonstrou a necessidade da utilização de 384 participantes, sendo 117 usuários de ESF e 267 usuários de atenção primária tradicional, levando em consideração a população cadastrada em cada unidade de saúde incluída no estudo.

Os dados foram coletados entre janeiro e junho de 2016 por intermédio da aplicação do instrumento Primary Care Assessment Tool (PCATool-Brasil) - usuários adultos, versão reduzida (quadro 1), a fim de otimizar o processo de aplicação e utilização dos resultados em ações estratégicas (OLIVEIRA ET AL., 2013). Esse instrumento é validado e está disponível gratuitamente. 
Quadro 1. Instrumento Primary Care Assessment Tool (PCATool-Brasil) - usuários adultos, versão reduzida

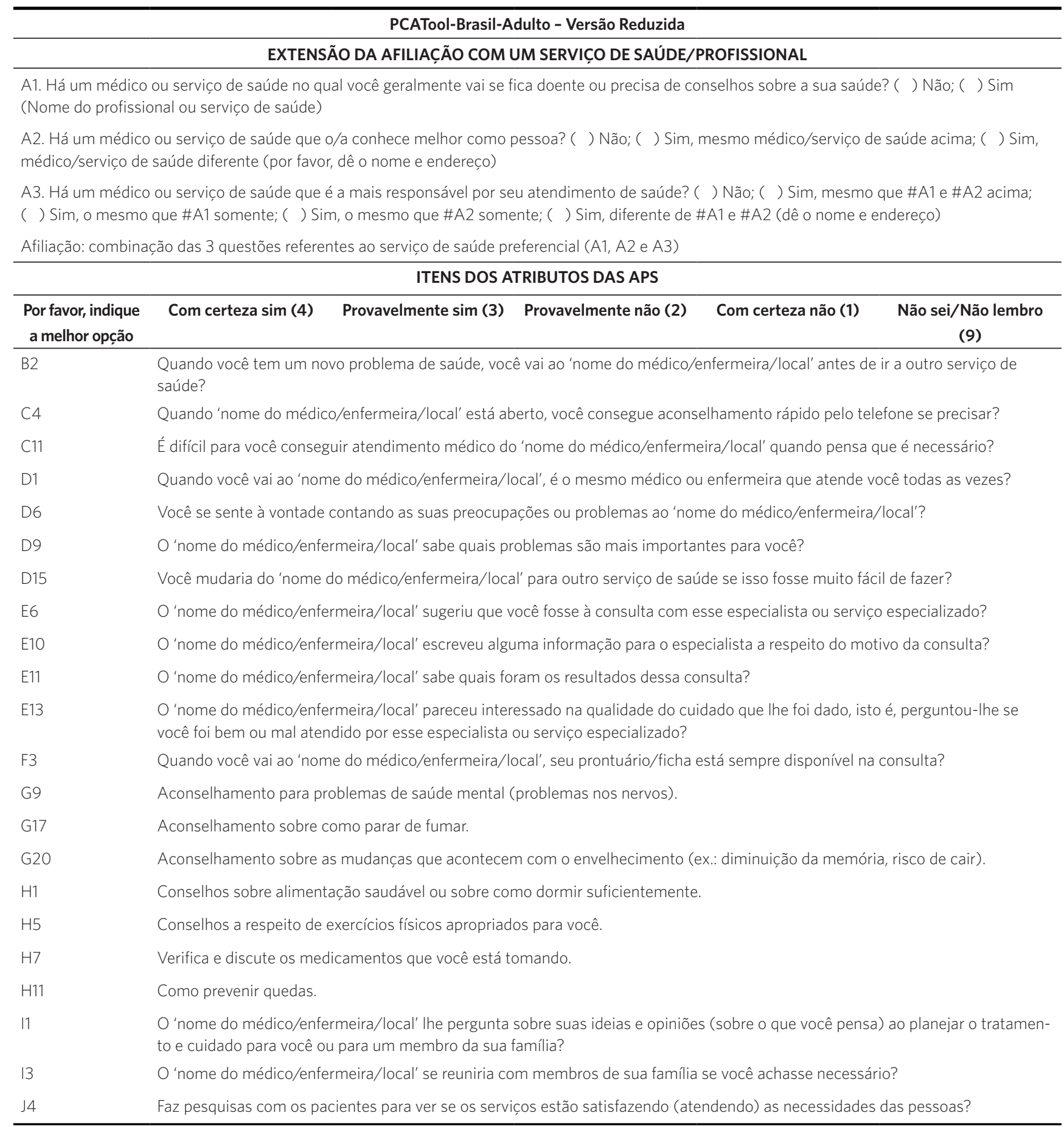


A versão reduzida do PCATool-Brasil é composta por 23 itens, contemplando os atributos essenciais: acesso de primeiro contato do indivíduo com o sistema de saúde (utilização e acesso); longitudinalidade; integralidade (serviços disponíveis e serviços prestados) e; coordenação (cuidado e sistema de informações); além de outros dois atributos derivados: orientação familiar e orientação comunitária (OLIVEIRA ET AL., 2013). Esse instrumento é baseado no modelo de avaliação da qualidade de serviços de saúde proposto por Donabedian e mede a presença e extensão dos atributos da APS por meio de escala Likert, com cinco opções de resposta: 'com certeza sim' (valor=4); 'provavelmente sim' (valor=3); 'provavelmente não' (valor=2); ‘com certeza não' (valor=1) e; 'não sei/não lembro' (valor=9), possibilitando construir escores para cada atributo ou seu componente separadamente (BRASIL, 2010).

Ainda, em relação aos usuários, foi aplicado um questionário a respeito do perfil sociodemográfico, contendo questões relativas ao sexo, idade, anos de estudo e situação conjugal dos participantes.

Foram entrevistados usuários com mais de 18 anos, que estiveram presentes nas unidades de saúde selecionadas em dia predeterminado, já atendidos em sua unidade de referência e que aceitaram participar do estudo. Todos os participantes assinaram o Termo de Consentimento Livre e Esclarecido, em conformidade com a legislação vigente. As entrevistas foram realizadas pelo pesquisador treinado e duraram em média 15 minutos.

A tabulação e análise dos dados foram realizadas com auxílio do software estatístico Statistical Package for Social Sciences (SPSS) 20.0. Para assegurar maior qualidade, realizou-se a dupla digitação com posterior comparação dos resultados. Para fins de análise, efetuou-se a inversão dos valores do item C11 do PCAToolBrasil para: (valor 4=1), (valor 3=2), (valor 2=3) e (valor 1=4), já que ele foi formulado de maneira que quanto maior o valor atribuído, menor é a orientação para APS. Ademais, para aqueles participantes em que a soma de respostas em branco ('missing') e com respostas '9' ('não sei/ não lembro') atingiram $50 \%$ ou mais do total de itens de um componente, o escore não foi calculado, e quando contrário, as respostas ' 9 ' ('não sei/não lembro') foram transformadas no valor '2' ('provavelmente não'). Essa transformação se faz necessária para pontuar negativamente algumas características do serviço de saúde que não são conhecidas pelo participante (BRASIL, 2010).

Em seguida, foram calculados escores referentes a cada um dos componentes relacionados com os atributos da APS presentes no PCATool-Brasil. Esses escores foram obtidos pela média aritmética simples dos valores das respostas dos itens que o compõe e transformados em escala de 0 a 10 utilizando da seguinte fórmula: (Escore obtido - 1) $\mathrm{x}$ 10/3 (BRASIL, 2010).

Logo, calcularam-se os escores Essencial e Geral da APS. O escore Essencial é medido pela soma do Grau de Afiliação mais escore médio de cada um dos componentes que pertencem aos atributos essenciais dividido pelo número de componentes. Já o escore Geral é medido pela soma do Grau de Afiliação mais escore médio dos componentes que pertencem aos atributos essenciais e derivados dividido pelo número total de componentes. Cabe ressaltar que o Grau de Afiliação não é considerado atributo da APS, mas é incluído no cálculo dos escores Essencial e Geral, e visa identificar o serviço ou profissional de saúde que serve como referência para os cuidados de saúde do entrevistado (BRASIL, 2010).

Consideraram-se altos escores os valores iguais ou maiores que 6,6, que equivalem a um ajustamento dos escores apontados na escala de Likert original a uma escala entre zero e dez, para cada um dos atributos avaliados, conforme recomendação de estudo que aplicam o mesmo instrumento (PAULA ET AL., 2015).

O presente estudo obedeceu aos preceitos éticos da Resolução no 466/2012 e foi aprovado pelo Comitê de Ética em Pesquisas da Universidade Federal de São João del-Rei CCO, sob parecer 1.234.728. 


\section{Resultados}

Participaram do estudo 384 usuários vinculados a 26 Uaps. Em relação ao perfil dos entrevistados, identificou-se que a maioria é do sexo feminino (80,7\%), 51,6\% são casados e $38,5 \%$ possuem menos de 4 anos de estudo (tabela 1). A idade média dos participantes é de 47,13 anos, variando entre 18 e 85 anos de idade.

Tabela 1. Perfil sociodemográfico dos participantes, segundo sexo, escolaridade e situação conjugal, 2016

\begin{tabular}{lcr}
\hline Variável & $\mathbf{n}$ & \% \\
\hline Sexo & 74 & 19,3 \\
\hline Masculino & 309 & 80,7 \\
\hline Feminino & & 38,5 \\
\hline Anos de Estudo & 148 & 27,6 \\
\hline$<4$ anos & 106 & 27,6 \\
De 4 a $<9$ anos & 106 & 6,3 \\
De 9 a 11 anos & 24 & 20,4 \\
12 anos ou mais & & 51,6 \\
\hline Situação conjugal & 78 & 6,0 \\
\hline Solteiro & 197 & 9,4 \\
Casado & 23 & 8,9 \\
União estável & 36 & 3,6 \\
Divorciado & 34 & 14 \\
Viúvo & & \\
Outros & &
\end{tabular}

Tabela 2. Valores médios e respectivos desvios padrões dos atributos da Atenção Primária à Saúde conferidos pelos usuários adultos na avaliação, em um município do centro-oeste de Minas Gerais (MG), 2016

\begin{tabular}{lcccrr}
\hline Atributos da APS & $\mathbf{n}$ & Mínimo & Máximo & Média & $\begin{array}{r}\text { Desvio } \\
\text { Padrão }\end{array}$ \\
\hline Grau de afiliação & 384 & 3,3 & 10,0 & 7,5 & 2,44 \\
Primeiro contato (Utilização) & 384 & 0,0 & 10,0 & 9,1 & 2,40 \\
Primeiro contato (Acessibilidade) & 327 & 0,0 & 10,0 & 6,0 & 3,40 \\
Longitudinalidade /Atendimento continuado & 383 & 0,0 & 10,0 & 5,7 & 1,92 \\
Coordenação (Cuidado e Sistemas de informação) & 208 & 3,3 & 10,0 & 7,2 & 1,60 \\
Integralidade (Serviços disponíveis) & 226 & 0,0 & 10,0 & 4,6 & 3,13 \\
Integralidade (Serviços prestados) & 376 & 0,0 & 10,0 & 5,0 & 3,20 \\
Enfoque familiar & 312 & 0,0 & 10,0 & 4,2 & 3,21 \\
Orientação comunitária & 301 & 0,0 & 10,0 & 1,9 & 3,50 \\
\hline
\end{tabular}


A tabela 2 apresenta os escores médios do Grau de Afiliação e dos atributos essenciais e derivados da APS analisados. Nela, identificou-se que o Grau de Afiliação e mais dois atributos (primeiro contato/utilização e coordenação/cuidado e sistemas de informação), ambos considerados essenciais, atingiram escore médio superior a 6,6, sendo assim bem avaliados. Os outros seis componentes relacionados com os atributos, quatro essenciais e dois derivados obtiveram escores inferiores a 6,6.

A partir da definição do Grau Afiliação e dos escores de cada um dos atributos, calcularam-se os escores Essencial e Geral. O escore Essencial alcançou média 6,5, variando entre 4,6 e 9,1; e o escore Geral atingiu 5,8, variando de 1,9 a 9,1, demonstrando, assim, que ambos não alcançaram a média mínima de 6,6.

\section{Discussão}

A avaliação da presença e extensão dos atributos da APS é fundamental para garantir seus resultados e a qualidade da assistência prestada à população, servindo de parâmetro para gestores, pesquisadores e profissionais (ARAÚJO, 2015; ARAújo ET AL., 2014). A avaliação dos atributos colabora para a reflexão acerca das práticas em saúde, promovendo a participação social e servindo como ferramenta de orientação às políticas de saúde e aos avanços no sistema local de saúde. Sendo considerada satisfatória, indica uma APS sólida, cujas ações de promoção, prevenção e recuperação da saúde são desenvolvidas de forma resolutiva, universal e distribuídas com equidade (MESQUITA FILHO; LUZ; ARAÚJO, 2014).

Em geral, os escores Essencial e Geral avaliados neste estudo não apresentaram índices satisfatórios. Destaca-se que o escore Essencial atingiu uma avaliação próxima ao desejável, podendo assim inferir que padrões mínimos esperados de orientação da APS foram alcançados. O valor do escore Essencial pode ter sido influenciado pelos altos escores obtidos pelo Grau de Afiliação e mais dois atributos considerados essenciais: primeiro contato (utilização) e coordenação (cuidado e sistemas de informação).

Já o baixo valor do escore Geral pode ser atribuído ao fato de que, no cálculo desse escore, são incluídos os atributos derivados, enfoque familiar e orientação comunitária, sendo estes os atributos que receberam os menores escores na avaliação. Outros estudos semelhantes, realizados em cenários diferentes, identificaram escores Essencial e Geral baixos, revelando que a prática dos serviços não está orientada por meio dos atributos da APS (ARAÚJO, 2015; ARAÚJO ET AL., 2014; HARZHEIM ET AL., 2016; SILVA; FRACOLLI, 2014).

No que diz respeito a cada um dos atributos da APS, a garantia do acesso de primeiro contato, em suas dimensões de utilização e acessibilidade, está relacionada com o uso da APS como a porta de entrada aberta e preferencial da rede de atenção, acolhimento dos usuários, promoção do vínculo e corresponsabilização pela atenção às suas necessidades de saúde e a capacidade da atenção primária em lidar e resolver diferentes problemas influenciados pelo contexto social (BRASIL, 2012; OLIVEIRA; PEREIRA, 2013; PAULA ET AL., 2016). A dimensão acessibilidade diz respeito à estrutura disponível; e a utilização refere-se aos processos estabelecidos no atendimento aos usuários.

Os resultados deste estudo mostram que a dimensão utilização obteve o maior escore entre todos os atributos avaliados, enquanto a dimensão acessibilidade obteve escore abaixo do desejado, demonstrando, assim, que o processo de trabalho das equipes em relação ao primeiro contato é muito bem avaliado enquanto a estrutura disponibilizada apresenta deficiências, comprometendo, dessa forma, o alcance pleno desse atributo. $\mathrm{O}$ atendimento pleno do acesso de primeiro contato pode contribuir para a redução da morbidade e mortalidade, de internação hospitalar, de tempo para a resolução do problema de saúde, de encaminhamentos 
desnecessários a especialistas e de custos totais. Resulta no uso eficiente de recursos, na atenção apropriada às necessidades e em melhores resultados em saúde (PAULA ET AL., 2016).

Evidencia-se que a disponibilidade à informação e gratuidade do serviço favorecem a dimensão utilização. Já fatores como linguagem e comunicação, dificuldade de encaminhamento a outros serviços, falta de confidencialidade e confiança, condições de saúde do usuário, características sociodemográficas, mudança frequente de residência, obrigações de emprego e responsabilidades familiares podem desfavorecer essa dimensão (PAULA ET AL., 2016).

O fato de a dimensão acessibilidade ter apresentado escore menor constitui-se um enorme desafio na busca da integralidade da atenção à saúde. Os usuários percebem o acesso ao serviço da APS como algo burocrático e demorado. Diante das dificuldades, os usuários tendem a buscar outras formas de conseguir atendimento a demandas que poderiam ser solucionadas na APS, sobrecarregando assim outros serviços (CAMPOS ET AL., 2014).

$\mathrm{O}$ acolhimento ou receptividade é peça importante na acessibilidade: uma boa recepção, a resolutividade, o ouvir o usuário, $o$ atender suas necessidades e a integralidade do cuidado são elementos fundamentais nesse processo. A adoção de ferramentas apropriadas de trabalho gerencial, tais como o horário de funcionamento, a oferta de contato telefônico, a presença de enfermeiro no local, o atendimento domiciliar, a abordagem multidisciplinar, o planejamento das ações, o equilíbrio entre atendimento da demanda espontânea e programada, a organização horizontal do trabalho e o compartilhamento do processo decisório, podem contribuir significativamente para oferecer a atenção ao primeiro contato (OLIVEIRA; PEREIRA, 2013; PAULA ET AL., 2016).

Outro atributo que recebeu avaliação positiva a partir da percepção dos usuários foi o de coordenação (cuidado e sistemas de informação). Esse atributo é considerado pilar da concepção estruturante e complexa da APS, em que pressupõe alguma forma de continuidade seja por parte do atendimento pelo mesmo profissional e do reconhecimento de problemas abordados em outros serviços, seja pela integração desse cuidado no cuidado global do paciente (BRASIL, 2010; SILVA; FRACOLLI, 2014). No entanto, pode-se afirmar que, sem a coordenação, a longitudinalidade diminuiria seu potencial, a integralidade seria comprometida e a função de primeiro contato teria conotação essencialmente administrativa (CARNEIRO ET AL., 2014).

Alguns fatores têm impacto sobre a coordenação do cuidado, entre eles: o incremento do papel dos médicos generalistas, relacionado com a gestão e com a responsabilização terapêutica do usuário na rede de atenção; o aumento da capacidade de resolução da APS, por meio da destinação de recursos e da ampliação de serviços ofertados; e a referência e contrarreferências bem estabelecidas. Reconhece-se que a desarticulação da rede ou o desconhecimento dos serviços de apoio disponíveis compromete a resolutividade da APS (CARNEIRO ET AL., 2014).

A avaliação do atributo longitudinalidade demonstra que os usuários desconhecem as unidades de saúde como fonte regular de cuidados, significando que a população não tem esses serviços como referência habitual para as suas necessidades de saúde. Autores sugerem que a falta de preparo dos profissionais de saúde ao lidar com a realidade das condições de vida e saúde da população, muitas vezes direcionando as atividades assistenciais para a doença, e não para a pessoa, ao número de atendimentos, e não para a qualidade da atenção, impedindo a formação de vínculos e serviço integral, constitui também um obstáculo ao alcance da longitudinalidade (OLIVEIRA; PEREIRA, 2013).

Observou-se a necessidade de estreitar a relação entre os profissionais de saúde e usuários, possibilitando diagnósticos mais precisos, tratamentos mais eficazes e principalmente o empoderamento dos indivíduos para o cuidado com a sua saúde, 
contribuindo, assim, para melhor resolutividade dos problemas de saúde. $\mathrm{O}$ atendimento a tal atributo só é possível se for uma prioridade da gestão, envolvendo as tecnologias de acolhimento, a oferta adequada de serviços, a fixação do profissional na unidade de saúde e, consequentemente, estabelecimento do vínculo e formação continuada (OLIVEIRA; PEREIRA, 2013; PAULA ET AL., 2016; SILVA; FRACOLLI, 2014).

$\mathrm{O}$ atributo integralidade (serviços disponíveis e serviços prestados), que inclui ações tanto do ponto de vista do caráter biopsicossocial do processo saúde-doença como ações de promoção, prevenção, cura/reabilitação e atenção em todos níveis de complexidade; alcançou escore abaixo da média esperada. A avaliação insatisfatória do atributo sugere a não abrangência do contexto do usuário e a descontinuidade assistencial. A atenção à saúde ainda se encontra centrada no modelo hospitalocêntrico, e como no sistema de saúde brasileiro coexistem os serviços públicos e privados, evidencia-se um confronto entre interesses e necessidades quando o usuário prioriza a busca de serviços com uma concentração tecnológica maior do que os da APS (MESQUITA FILHO; LUZ; ARAÚJO, 2014; OLIVEIRA; PEREIRA, 2013).

A integralidade exige que a APS reconheça a completa necessidade de saúde da população e disponibilize os recursos e estruturas necessárias para atendê-la (MESQUITA FILHO; LUZ; ARAÚJO, 2014). O propósito da integralidade imprime aos profissionais de saúde a tarefa de perceberem o usuário como sujeito histórico, social e político, articulado ao contexto familiar, ao meio ambiente e à sociedade na qual se insere (PAULA ET AL., 2016). Dessa forma, permite-se a elaboração de planos de cuidado que atendem às reais necessidades da população, construindo a possibilidade do cuidado centrado no usuário e contribuindo para a melhoria da qualidade dos serviços de saúde prestados (PAULA ET AL., 2016).

Nesse sentido, APS se orienta também por meio dos atributos derivados como o enfoque familiar e orientação comunitária.
Estes atributos envolvem a estimulação da participação dos usuários como forma de ampliar sua autonomia e capacidade na construção do cuidado à sua saúde e das pessoas e coletividades do território, no enfrentamento dos determinantes e condicionantes de saúde, na organização e orientação dos serviços de saúde a partir de lógicas mais centradas no usuário e no exercício do controle social (BRASIL, 2012).

Um serviço de saúde orientado para a APS com a presença de características como orientação familiar e comunitária permite que o cuidado seja mais efetivo, contribuindo para a melhoria dos indicadores de saúde. A clínica ampliada visa romper as barreiras com a clínica tradicional, modificando o processo de trabalho, partindo para o cuidado centrado nos sujeitos e no âmbito coletivo, modificando o modo de cuidar hegemônico, medicocêntrico e pautado nas ações programáticas (ARAÚJO ET AL., 2014).

Os dois atributos obtiveram os piores escores, sendo que o atributo orientação comunitária recebeu valor de 1,9 , considerado muito abaixo da média proposta. Outros estudos carreiam uma avaliação ruim desses mesmos atributos, demonstrando que o atributo enfoque familiar obteve o pior resultado (ARAÚJO ET AL., 2014; SILVA, FRACOLLI, 2014). Pressupõem-se que a avaliação negativa desses dois escores pode ser explicada, em parte, pelo número significativo de entrevistados que são usuários de Uaps tradicionais.

Isso pode revelar ainda o não reconhecimento, por parte do serviço, da epidemiologia prevalente e especificidades de saúde da população, da ausência de contato direto com a população adscrita, assim como a ausência de planejamento e avaliação, e da continuidade de uma atenção pautada no modelo individual e curativo (MESQUITA FILHO; LUZ; ARAÚJO, 2014).

$\mathrm{O}$ enfoque familiar ocorre quando os indivíduos são considerados dentro de seus ambientes, do contexto familiar e de seus recursos, além da sua exposição a ameaças à saúde. Ademais, uma abordagem orientada 
para a comunidade aplica os métodos da clínica, epidemiologia, ciências sociais, pesquisa e avaliação de serviços de saúde. Cabe ressaltar que o planejamento das práticas de saúde no cenário em estudo não envolve a participação da comunidade. É necessária, portanto, uma nova forma de organizar e agir em saúde com a constituição de saberes e de ações que se interpenetrem (OLIVEIRA; PEREIRA, 2013).

\section{Considerações finais}

Os atributos essenciais e derivados da APS analisados receberam avaliação heterogênea; e a maioria necessita de um olhar mais atento e de investimento por parte dos gestores e profissionais. Denota-se um enorme desafio na busca da integralidade da atenção à saúde e a necessidade de orientar as práticas de acordo com as necessidades da população e de dados epidemiológicos, por meio da construção do diagnóstico local e, consequentemente, planejamento de ações, elaboração e reformulação das políticas e programas e organização e aprimoramento da rede de serviços de saúde nos demais níveis assistenciais.

É necessária uma atenção maior acerca do enfoque familiar e orientação comunitária, da utilização de tecnologias de acolhimento, aquisição de confiança mútua e duradoura entre os usuários e os profissionais de saúde, além de maior sensibilização acerca da participação popular e do controle social.

Cabe ressaltar que o instrumento PCATool-Brasil - usuários adultos na versão reduzida - foi de fácil aplicação e manuseio. Sugere-se a sua utilização para uso rotineiro, a fim de avaliar a qualidade e orientação a APS, sob a percepção dos usuários.

\section{Referências}

ARAÚJO, L. U. A. et al. Avaliação da qualidade da atenção primária à saúde sob a perspectiva do idoso. Ciência \& Saúde Coletiva, Rio de Janeiro, v. 19, n. 8, p. 3521-3532, 2014. Disponível em: <http://www.scielo.br/ scielo.php?pid=S1413-81232014000803521\&script $=$ sci abstract\&tlng=pt>. Acesso em: 3 jan. 2017.

ARAÚJO, R. L.; MENDONÇA, A. V. M.; SOUSA, M.

F. Percepção dos usuários e profissionais de saúde no Distrito Federal: os atributos da atenção primária. Saúde em Debate, Rio de Janeiro, v. 39, n. 105, abr./jun. 2015. Disponível em: <http://www.scielo.br/pdf/sdeb/ v39n105/0103-1104-sdeb-39-105-00387.pdf>. Acesso em: 3 jan. 2017.
BRANDÃO, A. L. R. B. S.; GIOVANELLA, L.; CAMPOS, C. E. A. Avaliação da atenção básica pela perspectiva dos usuários: adaptação do instrumento EUROPEP para grandes centros urbanos brasileiros. Ciência \& Saúde Coletiva, Rio de Janeiro, v. 18, n. 1, p. 103-114, 2013. Disponível em: <http://www.scielo.br/scielo. php?pid=S1413-81232013000100012\&script $=$ sci abstract\&tlng=pt>. Acesso em: 3 jan. 2017.

BRASIL. Ministério da Saúde. E-Gestor Atenção Básica. 2017. Disponível em: <https://egestorab.saude.gov.br/ paginas/acessoPublico/relatorios/relHistoricoCoberturaAB.xhtml>. Acesso em: 16 nov. 2016. 
Ministério da Saúde. Secretaria de Atenção em Saúde. Departamento de Atenção Básica. Manual do instrumento de avaliação da atenção primária à saúde: primary care assessment tool pcatool - Brasil. Brasília, DF: Ministério da Saúde, 2010. Disponível em: <http:// bvsms.saude.gov.br/bvs/publicacoes/manual_avaliacao_pcatool_brasil.pdf>. Acesso em: 3 jan. 2017.

Ministério da Saúde. Secretaria de Atenção à Saúde. Departamento de Atenção Básica. Política Nacional de Atenção Básica. Brasília, DF: Ministério da Saúde, 2012. Disponível em: <http://189.28.128.100/ dab/docs/publicacoes/geral/pnab.pdf>. Acesso em: 16 nov. 2016

CAMPOS, R. T. O. et al. Avaliação da qualidade do acesso na atenção primária de uma grande cidade brasileira na perspectiva dos usuários. Saúde em Debate, Rio de Janeiro, v. 38, p. 252-264, out. 2014. Disponível em: <http://www.scielo.br/pdf/sdeb/v38nspe/0103-1104sdeb-38-spe-0252.pdf>. Acesso em: 16 nov. 2016.

CARNEIRO, M. S. M. et al. Avaliação do atributo coordenação da atenção primária à saúde: aplicação do PCATool a profissionais e usuários. Saúde em Debate, Rio de Janeiro, v. 38, out., 2014. Disponível em: <http://www.scielo.br/scielo.php?pid=S0103$-11042014000600279 \&$ script $=$ sci_abstract $\&$ tlng $=$ pt $>$. Acesso em: 16 nov. 2016

HARZHEIM, E. et al. Avaliação dos usuários crianças e adultos quanto ao grau de orientação para Atenção Primária à Saúde na cidade do Rio de Janeiro, Brasil. Ciência \& Saúde Coletiva, Rio de Janeiro, v. 21 n. 5, maio, 2016. Disponível em: <http:// www.scielo.br/scielo.php?script=sci_arttext\&pid $=$ S1413-81232016000501399> . Acesso em: 16 nov. 2016.

\section{INSTITUTO BRASLEIRO DE GEOGRAFIA E}

ESTATÍSTICA (IBGE). Divinópolis, Minas Gerais. 2016. Disponível em: <http://tabnet.datasus.gov.br/cgi/tabcgi.exe?ibge/cnv/poptMG.def>. Acesso em: 8 jun. 2016.

MESQUITA FILHO, M. M.; LUZ, B. S. R.; ARAÚJO, C. S. A Atenção Primária à Saúde e seus atributos: a situação das crianças menores de dois anos segundo suas cuidadoras. Ciência \& Saúde Coletiva, Rio de Janeiro, v.
19, n. 7, jul. 2014. Disponível em: <http://www.scielo.br/ scielo.php?pid=S1413-81232014000702033\&script=sci abstract\&tlng=pt >. Acesso em: 16 nov. 2017.

OLIVEIRA, M. A. C.; PEREIRA, I. C. Atributos Essenciais da Atenção Primária e a Estratégia Saúde da Família. Revista Brasileira de Enfermagem, Brasília, DF, v. 66, p. 158-64, 2013. Disponível em: <http://www. scielo.br/pdf/reben/v66nspe/v66nspea20.pdf>. Acesso em: 16 nov. 2016.

OLIVEIRA, M. M. C. et al. PCATool-ADULTOBRASIL: uma versão reduzida. Revista Brasileira de Medicina da Família e Comunidade. Rio de Janeiro, v. 8, n. 29, p. 256-63, out./dez., 2013. Disponível em: <https://rbmfc.org.br/rbmfc/article/view/823>. Acesso em: 16 nov. 2016.

PAIM, J. S. Atenção Primária à Saúde: uma receita para todas as estações? Saúde em Debate, Rio de Janeiro, v. 36, n. 94, p. 343-347, jul./set. 2012. Disponível em: <http://www.scielo.br/scielo.php?script=sci arttext\&pid=S0103-11042012000300004\&lng=en\&nrm =iso\&tlng=pt>. Acesso em: 16 nov. 2016.

PAULA, F. A. et al. Avaliação da atenção à saúde do adulto em um município-polo do Vale do Jequitinhonha (MG). Saúde em Debate, Rio de Janeiro, v. 39, n. 106, p. 802-814, jul./set. 2015. Disponível em: <http://www.scielo.br/pdf/sdeb/v39n106/0103-1104sdeb-39-106-00802.pdf>. Acesso em: 16 nov. 2016.

PAULA, C. C. et al. Fatores que interferem no acesso de primeiro contato na atenção primária à saúde: revisão integrativa. Revista de Pesquisa: Cuidado é Fundamental online, Rio de Janeiro, v. 8, n. 1, p. 40564078, jan./mar., 2016. Disponível em: <http://pesquisa. bvs.br/aps/resource/pt/bde-27386>. Acesso em: 16 nov. 2016.

SALA, A. et al. Integralidade e Atenção Primária à Saúde: avaliação na perspectiva dos usuários de unidades de saúde do município de São Paulo. Saúde e Sociedade, São Paulo, v. 20, n. 4, p. 948-960, 2011. Disponível em: <http://www.scielo.br/scielo. php?pid=S0104-12902011000400012\&script=sci abstract\&tlng=pt>. Acesso em: 16 nov. 2016. 
SILVA, C. S. O. et al. Integralidade e Atenção Primária à Saúde: avaliação sob a ótica dos usuários. Ciência \& Saúde Coletiva, Rio de Janeiro, v. 19, n. 11, p. 4407-4415, 2014. Disponível em: <http://www.scielosp.org/pdf/ csc/v19n11/1413-8123-csc-19-11-4407.pdf>. Acesso em 16 nov. 2016

SILVA, S. A.; FRACOLLI, L. A. Avaliação da Estratégia Saúde da Família: perspectiva dos usuários em Minas Gerais, Brasil. Saúde em Debate, Rio de Janeiro, v. 38, n. 103, p. 692-705, out./dez. 2014. Disponível em: <http://www.scielo.br/pdf/sdeb/v38n103/0103-1104sdeb-38-103-0692.pdf>. Acesso em: 16 nov. 2016.
STARFIELD, B.; XU, J.; SHI, L. Validating the Adult Primary Care Assessment Tool. The Journal of Family Practice, Nova Jersey, v. 50, n. 2, p.161-175, 2001.

Recebido para publicação em fevereiro de 2017

Versão final em julho de 2017

Conflito de interesses: inexistente

Suporte financeiro: não houve 